Received: $\quad 2018.10 .18$ Accepted: 2019.01.08 Published: 2019.04 .24

\title{
Role of Allelic Imbalance in Predicting Hepatocellular Carcinoma (HCC) Recurrence Risk After Liver Transplant
}

Authors' Contribution: Study Design A Data Collection B Statistical Analysis C Data Interpretation D Manuscript Preparation E Literature Search F Funds Collection G
ABCDEF 1 Duilio Pagano*

BD 2 Floriana Barbera*

AFG 2,3 Pier Giulio Conaldi

BD 1 Aurelio Seidita

BF 1 Fabrizio Di Francesco

B 2 Daniele Di Carlo

CD 1 Marco Barbàra

CD 4 Fabio Tuzzolino

AG 5 Angelo Luca

AF 1 Salvatore Gruttadauria
1 Department for Treatment and Study of Abdominal Diseases and Abdominal Transplantation, Mediterranean Institute for Transplantation and High Specialization Therapies (IRCCS-ISMETT), Palermo, Italy 2 Department of Laboratory Medicine and Advanced Biotechnologies, Mediterranean Institute for Transplantation and High Specialization Therapies (IRCCS-ISMETT), Palermo, Italy

3 Ri.MED Foundation, Palermo, Italy

4 Research Office, Mediterranean Institute for Transplantation and High

Specialization Therapies (IRCCS-ISMETT), Palermo, Italy

5 Department of Diagnostic and Therapeutic Services, Mediterranean Institute for Transplantation and High Specialization Therapies (IRCCS-ISMETT), Palermo, Italy
Corresponding Author: Source of support:
* Duilio Pagano and Floriana Barbera contributed equally to this study

Salvatore Gruttadauria, e-mail: sgruttadauria@ismett.edu

Departmental sources

Background: One of the most controversial problems for liver transplantation in patients affected by hepatocellular carcinoma (HCC) remains the lack of an oncologic staging system to predict cancer recurrence after liver transplantation (LT). We analyzed allelic imbalance (AI) in 19 microsatellites, and assessed the post-LT HCC recurrence risk.

Material/Methods: Seventy-one patients were included; 18 had tumor recurrence within 5 years post-transplant. Molecular analysis was done in the primary HCC and peripheral blood samples: a total of 19 microsatellites was used to assess Al. Specific Al was evaluated when outside of range value between 0.66 and 1.5. Based on data in the literature, we grouped the 19 microsatellites into 4 panels. We calculated the fractional allelic imbalance (FAI) to make comparisons between different panels including different subsets of microsatellites.

Results: We report that Al was associated with HCC recurrence in 3 main loci (D3S2303, D9S251, and D9S254). Tumor recurrence was associated only with 2 specific panels with 9 microsatellites previously reported to be associated with high risk for HCC recurrence. Our data show that fractional allelic imbalance (FAl) index has good negative ability to predict HCC recurrence (Panel 2: negative predictive value of $95 \%$ ).

Conclusions: Al analysis could have prognostic value in risk management of HCC recurrence after LT, especially for early recurrence.

MeSH Keywords: $\quad$ Allelic Imbalance • Carcinoma, Hepatocellular • Liver Transplantation • Treatment Outcome

Abbreviations:

AI - allelic imbalance; AR - allelic ratio; AUC - area under the ROC curve; CT - computerized tomography; FAI - fractional allelic imbalance; FFPE - formalin-fixed paraffin-embedded; HCC - hepatocellular carcinoma; LOH - loss of heterozygosis; LT - liver transplantation; MELD - model for end-stage liver disease; MRI - magnetic resonance imaging; NPV - negative predictive value; $\mathbf{O R}$ - odds ratio;

PPV - positive predictive value; RFS - recurrence-free survival; $\mathbf{R O C}$ - receiver operative characteristic;

TNM - tumor-node-metastasis

Full-text PDF: https://www.annalsoftransplantation.com/abstract/index/idArt/913692

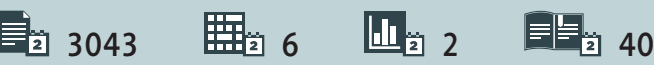




\section{Background}

Over the last 20 years hepatocellular carcinoma (HCC) has become one of the most common indications for liver transplantation (LT). Data from the European Liver Transplant Registry from 1988 to 2015 show that cancer represents $16.5 \%$ of overall causes of LT, and $87.3 \%$ are HCC-related. Survival analysis demonstrates that long-term survival ( 5 or more years after LT) of this specific subset of patients is lower than that of recipients with other diseases [1], as also reported by the Organ Procurement and Transplantation Network in the USA [2].

In a seminal paper published in 1997, Mazzaferro et al. established what came to be called the Milan criteria, an attempt to standardize criteria for transplantation of patients with HCC. They reported that patients with either 1 tumor with a diameter $\leq 5 \mathrm{~cm}$, or patients with $2 / 3$ tumors each with a diameter $\leq 3 \mathrm{~cm}$, had a recurrence-free survival (RFS) rate at 5 years of $75 \%$ and $83 \%$, respectively [3]. The Milan criteria have been validated by several groups, and were adopted by the United Network for Organ Sharing UNOS staging system for allocating organs, although the criteria have also been criticized for being too restrictive and for limiting the prognostic information to only radiologic appearance, without considering other clinical, molecular, or pathologic data [4]. Other research groups have tried to extend the Milan criteria, some based on dimensional features [5-8], while others have considered microvascular invasion $[9,10]$ or tumor grade [11], or evaluating biochemical parameters [12-14]. Mazzaferro et al. tried to simplify data, first with the Metroticket Paradigm [15], and then by proposing the Up-to-7 Criteria [16].

Nonetheless, the debate over the criteria for transplantation of HCC patients is still far from over. Some recent studies have defined loss of heterozygosity $(\mathrm{LOH})$ or allelic imbalance (Al) according to the level of gene damage and genomic instability, and explored whether a panel of tumor-suppressor gene markers could be useful in predicting recurrence with an index of cumulative mutational damage (fractional allelic imbalance, FAI) [17-19].

Other studies have reported that panels including specific microsatellites were significantly correlated with the post-transplant recurrence-free survival (RFS), but none achieved perfect discrimination alone [20,21], and proposed a rational approach using FAl in conjunction with models based on clinical data to expand the conventional LT selection policies [22].

Recently, Schmidt, and Marsh, at UPMC, proposed integrating data from FAl and macrovascular invasion, developing a novel staging system predicting HCC recurrence after LT (Pittsburgh staging system) [23].
Our study aimed to analyze the microsatellite LOHs to assess the risk of tumor recurrence after LT in recipients with HCC, in order to help move the field away from morphometric criteria to ones that are based on molecular information on tumor biology.

\section{Material and Methods}

\section{Patients}

The study population included 71 orthotopic LT recipients affected by HCC at IRCCS - ISMETT, in Palermo, Italy between 2006 and 2012. Severity of chronic liver disease was based on the Model for End-stage Liver Disease score (MELD). Histopathological findings were used to determine the tumor stage, classified by the tumor-node-metastasis (TNM) staging system. To monitor hepatic recurrence or distant metastasis, all patients were routinely followed up for at least 3 years with blood tests, magnetic resonance imaging (MRI), and/or computerized tomography (CT) scans. ISMETT's Institutional Research Review Board and local Ethics Committee approved the research protocol (approval number IRRB/26/11, signed by Mariolina Crisci, $5^{\text {th }}$ December 2016).

\section{Microsatellite analysis}

Genomic DNA was extracted from whole blood, formalin-fixed paraffin-embedded (FFPE), amplified using Type-it Microsatellite PCR kit (QIAgen), and separated by capillary electrophoresis on the 3500 Genetic Analyzer (ThermoFisher). A total of 19 microsatellites were used to assess Al, which included 9 loci previously reported as significantly associated with a high tumor recurrence risk after LT [18, 24], 6 loci that may be associated with recurrence but were excluded from the UPMC group [23], and an additional 4 loci suggested as indicative of recurrence [23].

\section{Identification of microsatellites panels}

Based on data in the literature, we grouped the analyzed microsatellites into 4 different panels (Table 1) to assess the association between $\mathrm{FAl}$ and the presence of tumor recurrence. Panel 1 (9 loci) included the 9 microsatellites previously reported as associated with a high risk for HCC recurrence [24]. Panel 2 (15 loci) included an additional 6 microsatellites that may be associated with HCC recurrence [20]. Panel 3 (13 loci) included, in addition to the previous 9 loci, another 4 microsatellites suggested to be indicative of $\mathrm{HCC}$ recurrence (personal communication by Dr. Marsh, from UPMC). Panel 4 (19 loci) included all selected microsatellites (Table 1). 
Table 1. Microsatellites and panels.

\begin{tabular}{|c|c|c|c|c|c|c|}
\hline Gene & Locus & Microsatellite & Panel 1 & Panel 2 & Panel 3 & Panel 4 \\
\hline L-myc & $1 p 33$ & D1S162 & & & $x$ & $x$ \\
\hline L-myc & $1 \mathrm{p} 34$ & MYCL.5NT & $x$ & $x$ & $x$ & $x$ \\
\hline L-myc & $1 \mathrm{p} 35$ & D1S1161 & & & $x$ & $x$ \\
\hline CMM & $1 p 36$ & D1S407 & $\mathrm{x}$ & $x$ & $\mathrm{x}$ & $\mathrm{x}$ \\
\hline OGG1 & $3 p 24$ & D3S2303 & & $x$ & & $x$ \\
\hline VHL & $3 p 26$ & D3S1539 & $x$ & $x$ & $x$ & $x$ \\
\hline APC & $5 q 21$ & D5S615 & $x$ & $x$ & $x$ & $x$ \\
\hline MCC & $5 p 21$ & D5S592 & & $x$ & & $x$ \\
\hline PTCH & $9 q 22$ & D9S252 & & $x$ & & $x$ \\
\hline CDKN2A/p16 & $9 p 21$ & D9S251 & $x$ & $\mathrm{x}$ & $x$ & $x$ \\
\hline CDKN2A/p16 & $9 p 21$ & D9S254 & & $\mathrm{x}$ & & $\mathrm{x}$ \\
\hline PTEN & $10 q 23$ & D10S520 & & $x$ & & $\mathrm{x}$ \\
\hline PTEN & $10 q 23$ & D101173 & & $x$ & & $x$ \\
\hline TP53 & $17 p 13$ & D17S1289 & $\mathrm{x}$ & $\mathrm{x}$ & $\mathrm{x}$ & $\mathrm{x}$ \\
\hline TP53 & $17 p 13$ & D17S974 & $x$ & $x$ & $x$ & $x$ \\
\hline TP53 & $17 p 13$ & TP53 L1 & $x$ & $x$ & $x$ & $\mathrm{x}$ \\
\hline TP53 & $17 p 23$ & D17S786 & & & $x$ & $\mathrm{x}$ \\
\hline TP53 & $17 p 23$ & D17S516 & & & $\mathrm{x}$ & $\mathrm{x}$ \\
\hline DCC/SMAD4 & $18 q 21$ & D18S814 & $x$ & $x$ & $x$ & $x$ \\
\hline
\end{tabular}

\section{Allelic imbalance analysis}

An Al analysis investigated and compared healthy tissue (peripheral blood samples) and diseased tissue in the primary HCC. Microsatellite markers were designated as informative/ heterozygous (2 different alleles/peaks) or non-informative/ homozygous (1 allele/peak excluded for frequency calculation of $\mathrm{Al}$ ). For any informative microsatellite, signal intensity in tumor DNA was compared with those of the corresponding normal DNA; the allele ratio (AR) between 2 allele peaks for each marker for each sample (peak height of allele 1/peak height of allele 2) was calculated, and Al was subsequently obtained as the ratio between the AR of the healthy sample and the AR of the diseased sample. Alleles were considered to be in balance when Al values were between 0.66 and 1.50 [21]. Two different grades of $\mathrm{LOH}$ were considered: 1) presence of $\mathrm{LOH}$ when Al values were either $0.50-0.65$ or $1.51-2.00$; 2) highlevel $\mathrm{LOH}$ when $\mathrm{Al}$ values were either below 0.50 or greater than 2.00 , respectively [18-29].

To make comparisons between different panels including different subsets of microsatellites, we calculated the FAl as the proportion of informative (heterozygous) microsatellites that exhibit LOH over the total number of informative loci.

\section{Statistical analysis}

Continuous variables are expressed as mean and inter-quartile range (IQR), while categorical variables are expressed as frequency and percentage. Differences in the rates of HCC recurrence were tested by Fisher's exact test or Pearson's chi-square test without Yates correction, as appropriate; odds ratio (OR) was also used to assess the relationships. Predictive performances of different microsatellite panels were evaluated in terms of negative predictive value (NPV), positive predictive value (PPV), sensitivity, and specificity; the best discriminating cut-off value (Youden's index) was established for the FAl. GraphPad Prism 6, MedCal, and EpiTools were used for all statistical analyses.

\section{Results}

\section{Clinical data and HCC recurrence}

Of the 71 patients, 59 (83\%) were male, and HCV infection was the main cause of liver disease. TNM classification of HCC was available in 52 patients. Forty-eight patients had multiple tumors involving 1 or both liver lobes, and 29 had 
Table 2. Clinical and pathological data: Association between clinical data and HCC recurrence. Fisher's exact test and Pearson's chisquare (chi-square without Yates correction) were used, as appropriate.

\begin{tabular}{|c|c|c|c|c|}
\hline Clinical and pathological data & Overall & No HCC Recurrence & HCC Recurrence & p-Value \\
\hline $\mathrm{n}$ & 71 & 53 & 18 & \\
\hline Male Gender, no. (\%) & $59(83.1)$ & $44(83.0)$ & $15(83.3)$ & 1.000 \\
\hline Age, median [IQR] & $59.00[53.5,63.0]$ & $59.0[52.0,63.0]$ & $59.0[56.0,61.8]$ & 0.706 \\
\hline HCC etiology, no (\%) & & & & 0.216 \\
\hline HBV & $14(19.7)$ & $13(24.5)$ & $1 \quad(5.6)$ & \\
\hline $\mathrm{HBV}+\mathrm{HCV}$ & $1 \quad(1.4)$ & $1 \quad(1.9)$ & $0 \quad(0.0)$ & \\
\hline $\mathrm{HCV}$ & $49(69.0)$ & $35(66.0)$ & $14(77.8)$ & \\
\hline Other & $\begin{array}{ll}7 & (9.9)\end{array}$ & $4 \quad(7.5)$ & $3(16.7)$ & \\
\hline MELD, median [IQR] & $12.00[9.0,15.0]$ & $12.00[9.0,15.0]$ & $11.5[9.0,14.8]$ & 0.801 \\
\hline HCC nodules, no. (\%) & & & & 0.128 \\
\hline 1 & $23(32.4)$ & $19(35.8)$ & $4(22.2)$ & \\
\hline 2 & $19(26.8)$ & $16(30.2)$ & $3(16.7)$ & \\
\hline $3+$ & $29(40.8)$ & $18(34.0)$ & $11(61.1)$ & \\
\hline Largest nodule size $(\mathrm{cm})$, median [IQR] & $2.50[2.0,3.5]$ & $2.5[1.7,3.4]$ & $2.7[2.2,3.8]$ & 0.331 \\
\hline Histologic grade G2-G3, no. (\%) & $31(43.7)$ & $23(43.4)$ & $8(44.4)$ & 0.938 \\
\hline Vascular invasion, no. (\%) & $29(40.8)$ & $20(37.7)$ & $9(50.0)$ & 0.360 \\
\hline TNM (\%) & & & & 0.047 \\
\hline $\mathrm{T} 1$ & $10(14.1)$ & $10(18.9)$ & $0 \quad(0.0)$ & \\
\hline $\mathrm{T} 2-\mathrm{T} 3$ & $42(59.2)$ & $32(60.4)$ & $10 \quad(55.6)$ & \\
\hline NA & $19(26.8)$ & 11 (20.8) & $8(44.4)$ & \\
\hline Milan = Out (\%) & $44(62.0)$ & $36(67.9)$ & $8(44.4)$ & 0.076 \\
\hline
\end{tabular}

microvascular invasion. Forty-four patients were classified as beyond the Milan criteria after histological examination of the explanted liver. Clinicopathological data are summarized in Table 2.

During the median follow-up period of 5.26 years, 18 patients (25\%) developed intrahepatic and/or extrahepatic HCC recurrence, with most of the lesions already detectable within the first 2 years. Sites of tumor recurrence were liver (2), lung (5), abdominal cavity (4), bone (2), and multiple sites, in addition to the liver (5) [25-27]. No significant difference was found in clinical parameters, except for TNM Stage (Table 2).

\section{Association between $\mathrm{Al}$ and $\mathrm{HCC}$ recurrence}

Based on data in the literature $[18,20,24]$, we decided to analyze 19 loci situated within or adjacent to specific genes of interest. We found the presence of $\mathrm{Al}$ in at least 1 locus in $90 \%$ of patients with HCC recurrence compared with $74.5 \%$ of patients without recurrence. Significant association was found for D3S2303 $(p=0.048)$ considering the presence of LOH (Table 3A), and D1S407 $(p=0.006)$ D9S251 $(p=0.02), D 1 S 162(p=0.005)$, D5S592 ( $p=0.005)$, D9S254 $(p=0.002)$ and D10S520 $(p=0.04)$ considering high-level LOH (Table 3B).

\section{Evaluation of specific panels and association with HCC recurrence}

Descriptive analysis of the different panels showed a relevant grade of informativeness in Panel 2 (Table 4). We made a diagnostic test evaluation of each panel to assess the association between $\mathrm{Al}$ and the risk of HCC recurrence. No association was found between $\mathrm{LOH}$ and $\mathrm{HCC}$ recurrence, whereas a significant association was found in all panels considering high-level LOH, and the Panel 2 performance was better than the others (Table 5). The high significance of Panel 2 is further strengthened and supported by FAI analysis, which proves that a high proportion of informative microsatellites exhibit $\mathrm{LOH}$ over the total number of informative loci in this panel (Table 5). 
Table 3. Univariate Cox models for microsatellites association with hepatocellular carcinoma recurrence. (A) Patients with loss of heterozygosis (LOH). (B) Patients with high-level loss of heterozygosis.

\begin{tabular}{|c|c|c|c|c|}
\hline (A) & \multicolumn{2}{|c|}{$\exp ($ coef) [confint] } & p & Code \\
\hline D1S407.LOH1 & 0.74 & {$[0.10,5.68]$} & 0.7740 & \\
\hline MYCL1.LOH1 & 1.92 & {$[0.55,6.70]$} & 0.3036 & \\
\hline D3S1539.LOH1 & 2.06 & {$[0.81,5.23]$} & 0.1273 & \\
\hline D5S615.LOH1 & 2.07 & {$[0.77,5.57]$} & 0.1493 & \\
\hline D9S251.LOH1 & 2.55 & {$[0.91,7.19]$} & 0.0763 & \\
\hline D17S1289.LOH1 & 0.88 & {$[0.25,3.17]$} & 0.8492 & \\
\hline D17S974.LOH1 & 0.62 & {$[0.08,4.72]$} & 0.6471 & \\
\hline TP53.LOH1 & 3.11 & {$[0.34,28.23]$} & 0.3136 & \\
\hline D18S814.LOH1 & 1.46 & {$[0.50,4.27]$} & 0.4916 & \\
\hline D1S162.LOH1 & 0.86 & {$[0.25,2.94]$} & 0.8066 & \\
\hline D1S1161.LOH1 & 1.00 & {$[0.33,3.07]$} & 0.9991 & \\
\hline D17S516.LOH1 & 0.00 & {$[0.00, \operatorname{lnf}]$} & 0.9988 & \\
\hline D17S786.LOH1 & 3.40 & {$[0.44,26.20]$} & 0.2407 & \\
\hline D3S2303.LOH1 & 6.45 & {$[1.77,23.55]$} & 0.0048 & ** \\
\hline D5S592.LOH1 & 3.33 & {$[0.43,25.79]$} & 0.2485 & \\
\hline D9S254.LOH1 & 1.77 & {$[0.55,5.77]$} & 0.3398 & \\
\hline D9S252.LOH1 & 1.28 & {$[0.29,5.73]$} & 0.7456 & \\
\hline D10S1173.LOH1 & 2.52 & {$[0.80,7.98]$} & 0.1157 & \\
\hline D10S520.LOH1 & 2.14 & {$[0.61,7.52]$} & 0.2355 & \\
\hline
\end{tabular}

\begin{tabular}{|c|c|c|c|}
\hline (B) & $\exp ($ coef) [confint] & $\mathbf{p}$ & Code \\
\hline D1S407.HighLOH1 & $49.50 \quad[3.10,791.37]$ & 0.0058 & ** \\
\hline D3S1539.HighLOH1 & $1.42[0.47,4.33]$ & 0.5329 & \\
\hline D5S615.HighLOH1 & {$[0.75,5.67]$} & 0.1630 & \\
\hline D9S251.HighLOH1 & $4.58 \quad[1.27,16.45]$ & 0.0198 & * \\
\hline D17S1289.HighLOH1 & $4.26 \quad[0.93,19.43]$ & 0.0612 & . \\
\hline D17S974.HighLOH1 & $1.31 \quad[0.17,9.96]$ & 0.7916 & \\
\hline TP53.HighLOH1 & $10.49 \quad[0.95,115.73]$ & 0.0550 & \\
\hline D18S814.HighLOH1 & {$[0.75,9.51]$} & 0.1286 & \\
\hline D1S162.HighLOH1 & $31.50 \quad[2.86,347.37]$ & 0.0049 & ** \\
\hline D1S1161.HighLOH1 & {$[0.68,8.23]$} & 0.1781 & \\
\hline D17S786.HighLOH1 & $\begin{array}{c}8159967418361.53 \\
{[0.00, \text { Inf }]}\end{array}$ & 0.9999 & \\
\hline D5S592.HighLOH1 & $53.50 \quad[3.35,855.32]$ & 0.0049 & ** \\
\hline D9S254.HighLOH1 & $8.61 \quad[2.24,33.07]$ & 0.0017 & $* *$ \\
\hline D10S1173.HighLOH1 & {$[0.50,29.31]$} & 0.1956 & \\
\hline D10S520.HighLOH1 & $3.78 \quad[1.07,13.37]$ & 0.0387 & * \\
\hline
\end{tabular}

Table 4. Descriptive analysis of microsatellites panels fractional allelic imbalance (FAI) and loss of heterozygosity (LOH).

\begin{tabular}{|c|c|c|c|c|}
\hline & Panel 1 & Panel 2 & Panel 3 & Panel 4 \\
\hline$n$ & 71 & 71 & 71 & 71 \\
\hline \multicolumn{5}{|l|}{ Informativeness } \\
\hline Median & 77.8 & 93.3 & 76.9 & 73.7 \\
\hline IQR & $66.7-77.8$ & $86.7-100.0$ & $61.5-76.9$ & $68.4-78.9$ \\
\hline Presence of LOH, n (\%) & $48(67.6)$ & $51(71.8)$ & $53(74.6)$ & $56(78.9)$ \\
\hline \multicolumn{5}{|l|}{ FAl } \\
\hline Mean & 0.19 & 0.13 & 0.18 & 0.16 \\
\hline Std. dev. & 0.18 & 0.12 & 0.15 & 0.14 \\
\hline Median & 0.17 & 0.12 & 0.17 & 0.13 \\
\hline $\mathrm{IQR}$ & $0.00-0.31$ & $0-0.18$ & $0.04-0.25$ & $0.07-0.21$ \\
\hline Range & $0.00-0.62$ & $0-0.53$ & $0-0.58$ & $0-0.67$ \\
\hline Presence of high-level LOH, no. (\%) & $31(43.7)$ & $34(47.9)$ & $31(43.7)$ & $34(47.9)$ \\
\hline \multicolumn{5}{|l|}{ High-level of FAI } \\
\hline Mean & 0.09 & 0.05 & 0.07 & 0.06 \\
\hline Std. dev. & 0.12 & 0.07 & 0.10 & 0.08 \\
\hline Median & 0.00 & 0.00 & 0.00 & 0.00 \\
\hline $\mathrm{IQR}$ & $0-0.14$ & $0-0.07$ & $0-0.11$ & $0-0.08$ \\
\hline Range & $0-0.5$ & $0-0.30$ & $0-0.42$ & $0-0.40$ \\
\hline
\end{tabular}


Table 5. Univariate Cox models to assess the association between Al and the risk of HCC recurrence.

\begin{tabular}{|c|c|c|c|c|}
\hline \multicolumn{5}{|c|}{ At least one microsatellite } \\
\hline & $\exp (\operatorname{coef})[$ confint] & $\mathbf{p}$ & Code & Concordance \\
\hline Panel 1 & $2.95[0.86,10.09]$ & 0.0841 & . & 0.5961 \\
\hline Panel 2 & $4.06[0.94,17.52]$ & 0.0602 & . & 0.6092 \\
\hline Panel 3 & $2.01[0.59,6.86]$ & 0.2663 & & 0.5552 \\
\hline Panel 4 & $2.69[0.62,11.59]$ & 0.1848 & & 0.5683 \\
\hline \multicolumn{5}{|c|}{ At least one microsatellite (high-level) } \\
\hline & $\exp ($ coef) [confint] & $\mathbf{p}$ & Code & Concordance \\
\hline Panel 1 & $2.79[1.11,7.01]$ & 0.0286 & * & 0.6282 \\
\hline Panel 2 & $4.12[1.49,11.35]$ & 0.0062 & ** & 0.672 \\
\hline Panel 3 & $2.79[1.11,7.01]$ & 0.0286 & * & 0.6282 \\
\hline Panel 4 & $4.12[1.49,11.35]$ & 0.0062 & ** & 0.672 \\
\hline \multicolumn{5}{|c|}{ Fractional allelic imbalance } \\
\hline & $\exp ($ coef) [confint] & $\mathbf{p}$ & Code & Concordance \\
\hline Panel $1 \mathrm{FAl}$ & $11.64[1.11,122.15]$ & 0.0407 & * & 0.6379 \\
\hline Panel 2 FAI & $127.58[4.60,3535.44]$ & 0.0042 & ** & 0.6737 \\
\hline Panel 3 FAI & $10.04[0.70,143.33]$ & 0.0891 & . & 0.6172 \\
\hline Panel 4 FAl & $31.82[2.08,487.06]$ & 0.0129 & * & 0.6627 \\
\hline \multicolumn{5}{|c|}{ Fractional allelic imbalance (high-level) } \\
\hline & $\exp ($ coef) [confint] & p & Code & Concordance \\
\hline Panel 1 FAl & $148.58[9.39,2351.75]$ & 0.0004 & $* * *$ & 0.6825 \\
\hline Panel 2 FAI & $12736.03[192.99,840485.78]$ & $<0.0001$ & *** & 0.7255 \\
\hline Panel 3 FAI & $1784.22[40.74,78135.33]$ & 0.0001 & *** & 0.6914 \\
\hline Panel 4 FAl & $16309.40[298.76,890346.50]$ & $<0.0001$ & $* * *$ & 0.7395 \\
\hline
\end{tabular}

\section{Association of Al and time to HCC recurrence}

After proving the possible association between Al and HCC recurrence, we focused our attention on time to recurrence. Kaplan-Meier curves were performed, proving that $\mathrm{LOH}$, as expected, has no significant correlation with time to recurrence, whereas high-level LOH is strongly correlated with particularly high performance (Figure 1).

\section{Association of frequency of Al with early HCC recurrence}

To further extend our analysis, we assessed whether Al could have a prognostic value in early HCC recurrence detection. Thus, we focused our attention on the 14 patients (11 of whom were classified beyond the Milan criteria after pathology examination) with recurrence within 2 years of LT. We found that the FAl index for high-level LOH has good predictive negative performance. In particular, Panel 2 reaches a negative predictive value (NPV) of $95 \%$ due to its high sensitivity, with a loss of specificity and positive predictive value (PPV) lower than $35 \%$ (Table 6). This was confirmed by receiver operating characteristic (ROC) curve analysis (Figure 2).

\section{Association of Al with other predictors of HCC recurrence}

Finally, we focused our attention on any possible correlation between $\mathrm{Al}$ and other clinical or pathological parameters available in our dataset that could be associated with HCC recurrence [5-16]. We evaluated the association between $\mathrm{Al}$ and 3 different poor-prognosis HCC indicators: G2/G3 grading, vascular invasion, and Milan criteria. No single microsatellite showed any association with these 3 variables, neither considering presence of $\mathrm{LOH}$ nor considering high-level $\mathrm{LOH}$.

As previously reported, 31 patients were graded as having G2/G3 HCC; of these, 18 were graded G2, only 1 was graded G3, 


\section{A}

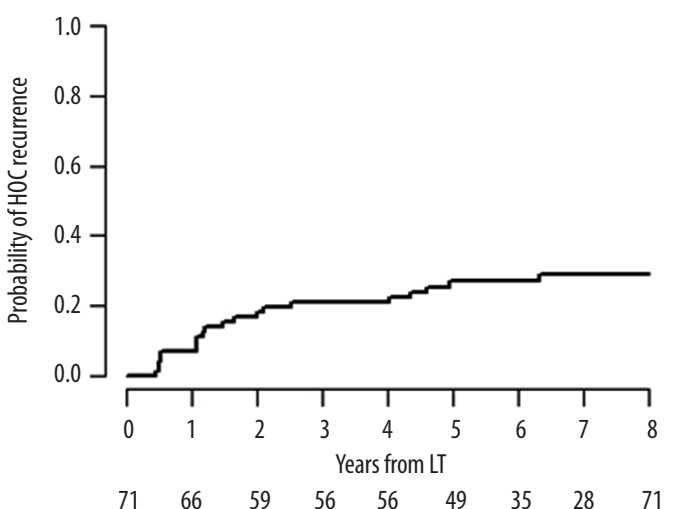

B
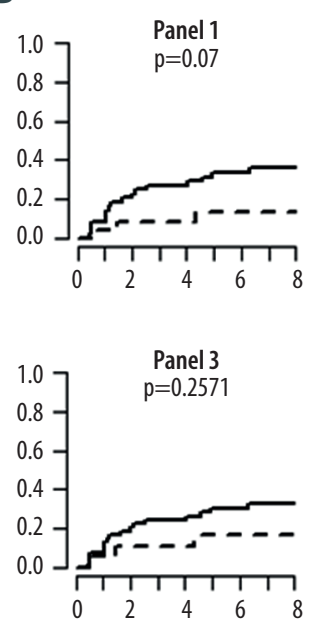

C
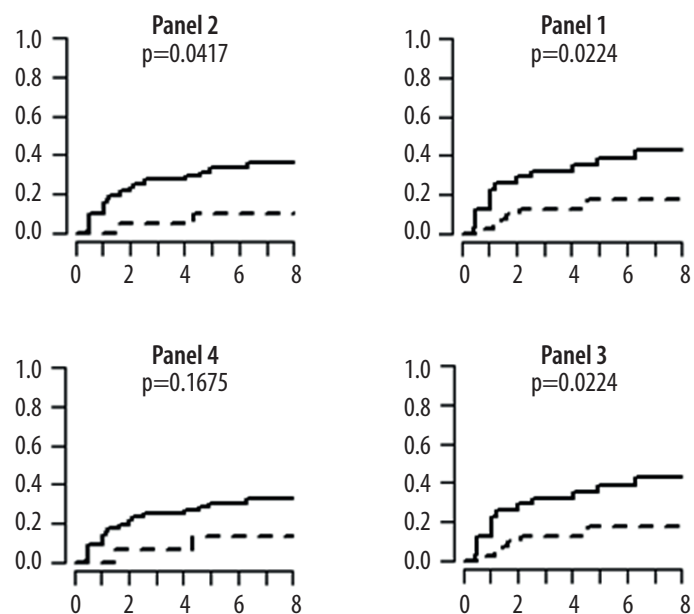
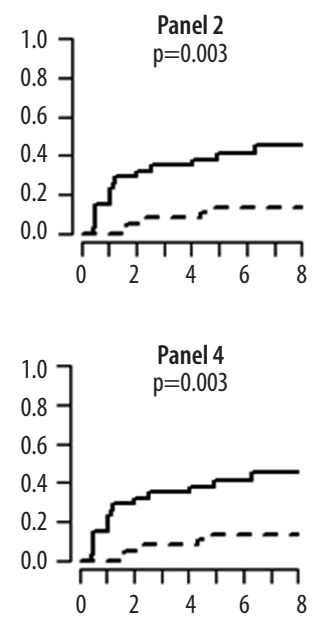

Figure 1. Kaplan-Meier time to recurrence curves. (A) Overall study population. (B) Patients with loss of heterozygosis. (C) Patients with high-level loss of heterozygosis.

and the remaining 12 were labelled G2-G3 by the pathologist. Importantly, only 5 of the 13 highest graded $(\mathrm{G} 3+\mathrm{G} 2-\mathrm{G} 3)$ were inside the Milan criteria, and only 1 of these 5 had an early recurrence. Only Panel 2 and Panel 4 succeeded in detecting this recurrence, considering both presence of $\mathrm{LOH}(\mathrm{Sp}=0.5, \mathrm{Se}=1$, $P P V=0.3, N P V=1)$ and high-level $\mathrm{LOH}(\mathrm{Sp}=0.75, \mathrm{Se}=1, \mathrm{PPV}=0.5$, $N P V=1)$, thus confirming in this small subset the performance observed in the overall study population.

\section{Discussion}

HCC recurrence after LT is one of the most important causes of mortality and morbidity [28]. At present, there are conflicting recommendations about what tumor characteristics are responsible for HCC recurrence, and the clinical and radiological criteria (e.g., Milan criteria, Up-to-7 criteria) seem to be inadequate because they provide characteristics of the tumor that do not always correspond to the actual pathological characteristics [16]. Several studies have identified microvascular invasion as the strongest independent predictor factor of recurrence $[29,30]$, associating it with other poor prognostic factors that, except for AFP, can only be known on histological analysis of the explanted liver, or need procedures that are invasive, not easily available, and lack adequate accuracy (e.g., liver biopsy) [31]. Scores based exclusively on radiological or biochemical parameters are not adequate to establish the risk of HCC recurrence, and a number of authors have attempted to combine the 2 methods. Mazzaferro et al. redesigned the Metroticket Paradigm [15], in which patients are assessed on the basis of the combination of AFP levels, and number and size of nodules [32]. A Belgian research group developed a model to predict the likelihood of recurrence based on 4 parameters: Time, Radiological-response, AFP, and INflammation (TRAIN) [33]. Similarly, the MoRAL (Model of Recurrence After Liver transplantation) score combines the biochemical features of AFP and neutrophil-to-lymphocyte ratio with the radiological feature of tumor dimension (tumor 
Table 6. Predictive performances of early recurrence panels.

\begin{tabular}{|c|c|c|c|c|c|}
\hline \multicolumn{6}{|c|}{ Loss of heterozygosis } \\
\hline & $\mathrm{Se}$ & Sp & PPV & NPV & Acc \\
\hline Panel 1 FAl & 0.8462 & 0.3621 & 0.2292 & 0.913 & 0.4507 \\
\hline Panel 2 FAl & 0.9231 & 0.3276 & 0.2353 & 0.95 & 0.4366 \\
\hline Panel 3 FAl & 0.8462 & 0.2759 & 0.2075 & 0.8889 & 0.3803 \\
\hline Panel 4 FAl & 0.9231 & 0.2414 & 0.2143 & 0.9333 & 0.3662 \\
\hline \multicolumn{6}{|c|}{ High-level loss of heterozygosis } \\
\hline & $\mathrm{Se}$ & Sp & PPV & NPV & Acc \\
\hline Panel $1 \mathrm{FAl}$ & 0.6923 & 0.6207 & 0.2903 & 0.9 & 0.6338 \\
\hline Panel 2 FAl & 0.8462 & 0.6034 & 0.3235 & 0.9459 & 0.6479 \\
\hline Panel 3 FAl & 0.6923 & 0.6207 & 0.2903 & 0.9 & 0.6338 \\
\hline Panel 4 FAl & 0.8462 & 0.6034 & 0.3235 & 0.9459 & 0.6479 \\
\hline
\end{tabular}

size $\geq 3 \mathrm{~cm}$ ) [34]. In the present retrospective study, we evaluated the relationship between prognostic clinicopathological factors and HCC recurrence, but no statistically significant association was found except for TNM stage (Table 2; $p=0.047$ ).

To date, however, it has not been possible to identify sufficiently reliable markers of the biological behavior of HCC. Therefore, the attention of researchers is increasingly shifting from the analysis of clinical and radiological characteristics to the analysis of genetic mutations typical of this neoplasm. In this context, several studies in the last few years have mentioned $\mathrm{Al}(\mathrm{LOH})$ as a possible predictor of HCC recurrence $[18,20,21,24]$. Marsh et al. developed a multivariate model (artificial neural network - ANN) based on 5 risk factors (sex, tumor number, tumor size, lobar tumor distribution, and grade of vascular invasion) that could predict the risk of tumor recurrence [35]. This model, in association with the study of Al based on tissue microdissection genotyping in 9 microsatellites at 6 genomic loci $[18,20]$, had an accuracy of $100 \%$, with a discriminatory power of $85 \%$ in the predicted 3-year recurrence outcome [36]. In addition, a recent meta-analysis of 41 eligible studies noted the importance of identifying serological (pre-LT AFP and pre-LT DCP) and molecular biomarkers able to predict HCC recurrence [37]. In our study, we found a significant association between Al in specific microsatellite loci and the risk of HCC recurrence, validating for the first time in a European population the results of several other studies $[18,20,22,24,35]$. A statistically significant association with tumor recurrence was found for D3S2303 (gene OGG1; $p=0.048$ ) considering the presence of $\mathrm{LOH}$ (Table $3 \mathrm{~A}$ ), and D1S407 (gene CMM; $p=0.006$ ) D9S251 (gene CDKN2A/p16; $p=0.02$ ), D1S162 (gene L-myc; $p=0.005$ ), D5S592 (gene MCC; $p=0.005$ ), D9S254 (gene CDKN2A/p16; $p=0.002$ ), and D10S520 (gene
PTEN; $p=0.04$ ) considering high-level LOH. Thus, we confirmed that the LOH frequency on 9p21 locus (D9S251 and D9S254, CDKN2A gene) is associated with recurrence [21].

Many retrospective studies have used different and arbitrary cumulative mutational damage indexes (e.g., FAl) to investigate the relationship between $\mathrm{Al}$ and HCC recurrence, and identified specific discriminatory values between true positives and false positives using ROC curve analysis constructed using results obtained in each study [18,20-23]. Our data show that the FAI index for high-level LOH in a specific panel of microsatellites (Panel 2) has a good negative predictive performance, with a 95\% NPV, to identify early-HCC recurrence. Unfortunately, the PPV for each analyzed panel cannot be considered satisfactory. Thus, our analysis confirms the existence of an association between HCC recurrence and LOH in specific loci (specifically high-level LOH), proving at the same time that Al should not be considered as a positive predictor of tumor recurrence, but its absence can be considered as a negative one.

Of note, in contrast to previously research, our study did not analyze the Al between neoplasia and healthy fragments of liver parenchyma, but obtained similar results by comparing very limited quantities of neoplastic tissue with whole blood, thus reducing the potential invasiveness of the analysis. In fact, previous studies have indicated that the use of minimally invasive laparoscopic techniques allows the successful resection of HCC nodules without increasing the risk of hepatic failure or peri-operative complications $[38,39]$. Thus, such surgical approaches could be proposed not only as a bridge-therapy for LT, but also as a useful diagnostic tool to evaluate Al between neoplastic tissue and whole blood [40]. The scenarios that would lead to a similar combined 
A
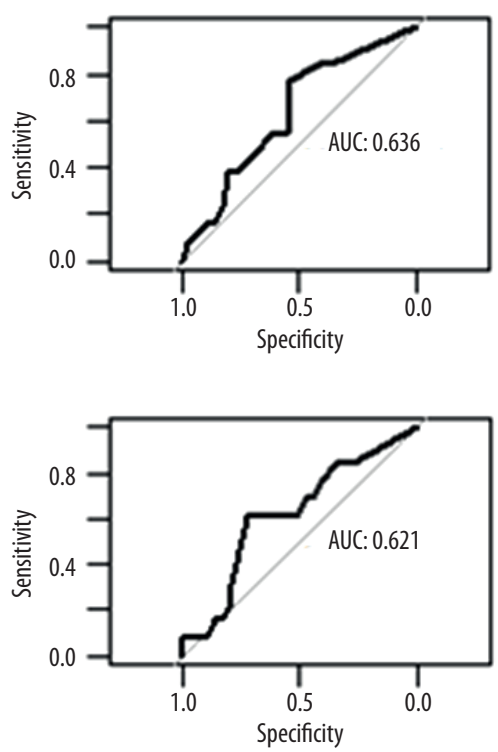

B
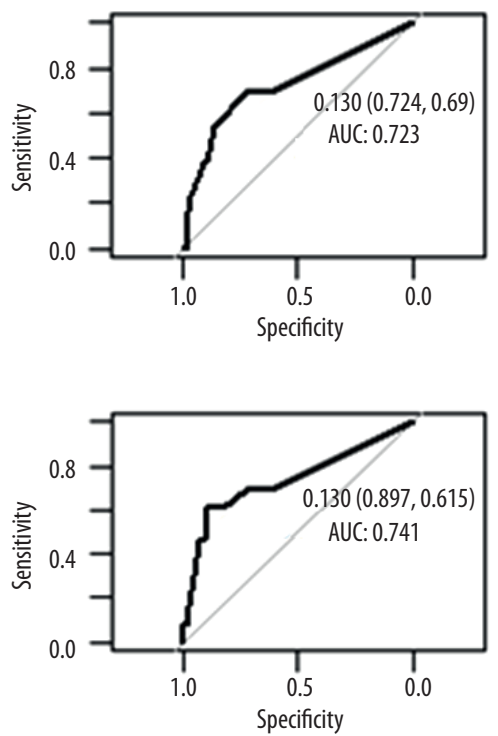
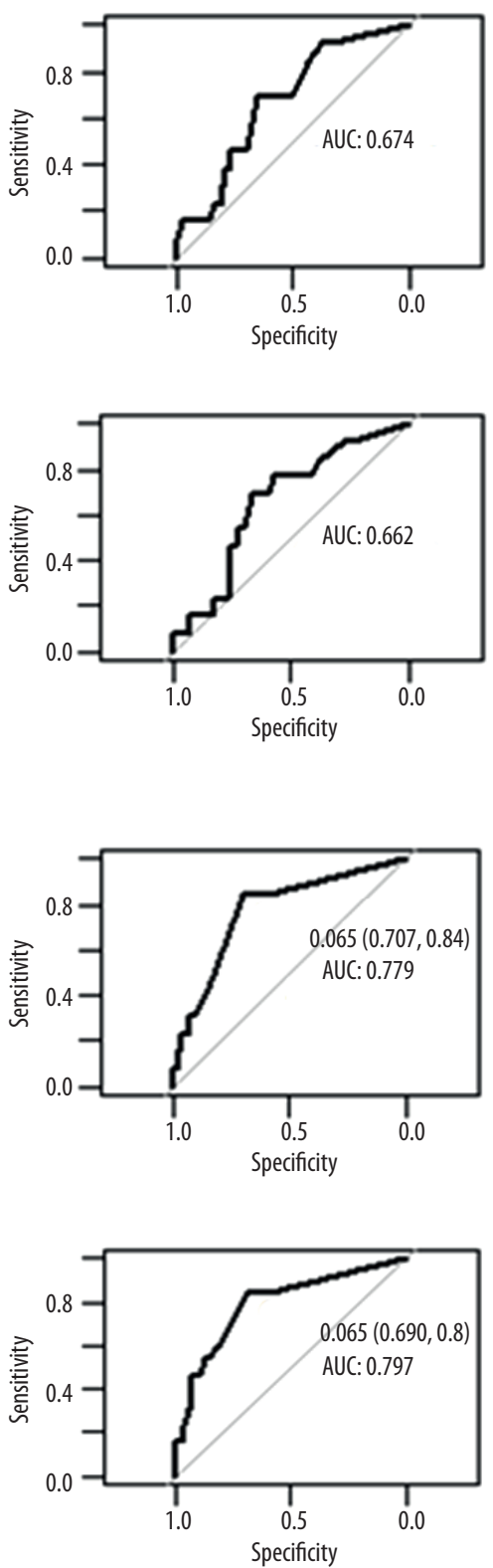

Figure 2. ROC curve for prediction of early recurrence. (A) Patients with loss of heterozygosis. (B) Patients with high-level loss of heterozygosis.

use of minimally invasive surgery and Al to predict patients at greater risk of recurrence could change the priorities in organ allocation procedures and would ensure tailored post-transplant therapies (e.g., changes of immunosuppression regimens).

Our study has several limitations. This was an analysis with a limited number of patients, and we could not evaluate the effect of pre-transplantation treatments on survival and recurrence (e.g., radiofrequency thermal ablation and trans-arterial chemoembolization). Unfortunately, there was no donor and recipient allelotyping, and we could not distinguish whether the tumor recurrence represents metastasis of the first cancer or a donor-derived de novo cancer, as proposed by recent studies [25-27]. Moreover, HCC genotyping can be complicated by interpretative difficulties due to heterogeneity of tumor tissues from a pathological specimen containing various subpopulations of cells. Unfortunately, we could not perform microdissection, and DNA from nonmalignant cells could have contaminated the DNA extracted from these samples. Finally, working with FFPE tissues of the native liver, and DNA extraction and amplification might be affected by tissue fixation time and progressive DNA degradation in archival tissue blocks. 
In the post-LT setting, FAl could provide useful information to adapt and personalize therapies (e.g., immunosuppression protocols), thus reducing the risk of recurrence. However, the application of molecular markers needs further evaluation before being used as selection criteria for LT. We intend to validate the HCC recurrence prediction model based on the use of specific microsatellites (especially D9S251) in a future study in order to verify its concrete predictive ability and to identify patients at lower risk of HCC recurrence, combining clinicopathological, radiological, and genomic data, easily available in the pre-LT setting. Moreover, using a larger number of patients with more complete clinical information, we intend to study the association of Al in each locus with clinicopathological characteristics and to analyze its biological role in HCC development, progression, and aggressiveness.

\section{Conclusions}

One of the important ethical implications of LT for HCC, which is the subject of lively debate in the transplantation community and beyond, is that ability to predict which recipients will

\section{References:}

1. European Liver Transplant Registry. Available: http://www.eltr.org/Overallindication-and-results.html

2. Organ Procurement and Transplantation Network. Available: https://optn. transplant.hrsa.gov/data/view-data-reports/national-data/

3. Mazzaferro V, Regalia E, Doci R et al: Liver transplantation for the treatment of small hepatocellular carcinomas in patients with cirrhosis. N Eng J Med, 1996; 334: 693-99

4. Marsh JW, Schmidt C: The Milan criteria: no room on the metro for the king? Liver Transpl, 2010; 16: 252-55

5. Yao FY, Ferrell L, Bass NM et al: Liver transplantation for hepatocellular carcinoma: Expansion of the tumor size limits does not adversely impact survival. Hepatology, 2001; 33: 1394-403

6. Herrero JI, Sangro B, Quiroga J et al: Influence of tumor characteristics on the outcome of liver transplantation among patients with liver cirrhosis and hepatocellular carcinoma. Liver Transpl. 2001;7: 631-36

7. Merli M, Nicolini G, Gentili F et al: Predictive factors of outcome after liver transplantation in patients with cirrhosis and hepatocellular carcinoma. Transplant Proc, 2005; 37: 2535-40

8. Ito T, Takada $\mathrm{Y}$, Ueda $\mathrm{M}$ et al: Expansion of selection criteria for patients with hepatocellular carcinoma in living donor liver transplantation. Liver Transpl, 2007; 13: 1637-44

9. Lai Q, Merli M, Ginanni Corradini S et al: Predictive factors of recurrence of hepatocellular carcinoma after liver transplantation: A multivariate analysis. Transplant Proc, 2009; 41: 1306-9

10. Marsh JW, Dvorchik I, Bonham CA, Iwatsuki S: Is the pathologic TNM staging system for patients with hepatoma predictive of outcome? Cancer, 2000 88: $538-43$

11. Cillo $U$, Vitale A, Bassanello $M$ et al: Liver transplantation for the treatment of moderately or well-differentiated hepatocellular carcinoma. Ann Surg, 2004; 239: 150-59

12. Yang SH, Suh KS, Lee HW et al: A revised scoring system utilizing serum alphafetoprotein levels to expand candidates for living donor transplantation in hepatocellular carcinoma. Surgery, 2007; 141: 598-609

13. Cillo $U$, Navaglia F, Vitale $A$ et al: Clinical significance of alpha-fetoprotein mRNA in blood of patients with hepatocellular carcinoma. Clin Chim Acta, 2004; 347: 129-38 successfully complete the post-transplant course. Transparency and clinical clarity are 2 of the cornerstones in sharing experiences with the medical community at large. We observed that the FAI for a high-level LOH has a good NPV for tumor recurrence within 2 years after transplant (95\%). These data confirm a relevant role of the CDKN2A gene for HCC recurrence. Our data suggest that the information obtained by $\mathrm{Al}$ analysis can be useful, and has a prognostic application in risk management of HCC recurrence in patients who have undergone LT, especially in early tumor recurrence. One of the important ethical implications of LT for HCC, which is the subject of lively debate in the transplantation community and beyond, is the ability to predict which recipients will successfully complete the post-transplant course. Developing new protocols for immunosuppressive regimen and surgical decision-making is paramount for continued success in this delicate field of medicine.

\section{Acknowledgments}

The authors would like to thank Warren Blumberg, of ISMETT's Language Services Department, for help in editing this paper.

14. Cillo U, Vitale A, Navaglia F et al: Role of blood AFP mRNA and tumor grade in the preoperative prognostic evaluation of patients with hepatocellular carcinoma. World J Gastroenterol, 2005; 11: 6920-25

15. Mazzaferro V: Results of liver transplantation: With or without Milan criteria? Liver Transpl, 2007; 13: S44-47

16. Mazzaferro V, Llovet JM, Miceli R et al., Metroticket Investigator Study Group Predicting survival after liver transplantation in patients with hepatocellular carcinoma beyond the Milan criteria: A retrospective, exploratory analysis. Lancet Oncol, 2009; 10: 35-43

17. Thorgeirsson SS, Grisham JW: Molecular pathogenesis of human hepatocellular carcinoma. Nat Genet, 2002; 31: 339-46

18. Marsh JW, Finkelstein SD, Demetris AJ et al: Genotyping of hepatocellular carcinoma in liver transplant recipients adds predictive power for determining recurrence-free survival. Liver Transpl, 2003; 9: 664-71

19. Woo HG, Park ES, Thorgeirsson SS, Kim YJ: Exploring genomic profiles of hepatocellular carcinoma. Mol Carcinog, 2011; 50: 235-43

20. Finkelstein SD, Marsh W, Demetris AJ et al: Microdissection-based allelotyping discriminates de novo tumor from intrahepatic spread in hepatocellular carcinoma. Hepatology. 2003; 37: 871-79

21. Schwartz M, Dvorchik I, Roayaie S et al: Liver transplantation for hepatocellular carcinoma: extension of indications based on molecular markers. J Hepatol, 2008; 49: 581-88

22. Dvorchik I, Schwartz M, Fiel MI et al: Fractional allelic imbalance could allow for the development of an equitable transplant selection policy for patients with hepatocellular carcinoma. Liver Transpl, 2008; 14: 443-50

23. Schmidt C, Marsh JW: Molecular signature for HCC: Role in predicting outcomes after liver transplant and selection for potential adjuvant treatment. Curr Opin Organ Transplant, 2010; 15: 277-82

24. Marsh JW, Dvorchik I: Liver organ allocation for hepatocellular carcinoma: Are we sure? Liver Transpl. 2003;9: 693-93

25. Altimari A, Gruppioni E, Fiorentino $M$ et al: Genomic allelotyping for distinction of recurrent and de novo hepatocellular carcinoma after orthotopic liver transplantation. Diagn Mol Pathol, 2005; 14: 34-38

26. Morita K, Taketomi A, Soejima $Y$ et al: De novo hepatocellular carcinoma in a liver graft with sustained hepatitis $C$ virus clearance after living donor liver transplantation. Liver Transpl, 2009; 15: 1412-16 
27. Vernadakis S, Poetsch M, Weber F et al: Donor origin de novo HCC in a noncirrhotic liver allograft 3 years after liver transplantation. Transpl Int, 2010; 23: $341-43$

28. Zavaglia C, De Carlis L, Alberti AB et al: Predictors of long-term survival after liver transplantation for hepatocellular carcinoma. Am J Gastroenterol. 2005; 100: 2708-16

29. Yao FY: Selection criteria for liver transplantation in patients with hepatocellular carcinoma: Beyond tumor size and number? Liver Transpl, 2006; 12: 1189-91

30. Dudek K, Kornasiewicz O, Remiszewski P et al: Impact of tumor characteristic on the outcome of liver transplantation in patients with hepatocellular carcinoma. Transplant Proc, 2009; 41: 3135-37

31. Takamori R, Wong LL, Dang C, Wong L: Needle-tract implantation from hepatocellular cancer: Is needle biopsy of the liver always necessary? Liver Transpl, 2000; 6: 67-72

32. Mazzaferro V, Sposito C, Zhou J et al: Metroticket 2.0 model for analysis of competing risks of death after liver transplantation for hepatocellular carcinoma. Gastroenterology. 2018;154: 128-39

33. Lai $Q$, Nicolini $D$, Inostroza Nunez $M$ et al: A novel prognostic index in patients with hepatocellular cancer waiting for liver transplantation: TimeRadiological-response-Alpha-fetoprotein-INflammation (TRAIN) score. Ann Surg, 2016; 264: 787-96
34. Halazun KJ, Najjar M, Abdelmessih RM et al: Recurrence after liver transplantation for hepatocellular carcinoma: A new MORAL to the story. Ann Surg, 2017; 265: 557-64

35. Marsh JW, Dvorchik I, Subotin M et al: The prediction of risk of recurrence and time to recurrence of hepatocellular carcinoma after orthotopic liver transplantation: A pilot study. Hepatology, 1997; 26: 444-50

36. Rodriguez-Luna H, Vargas HE, Byrne T, Rakela J: Artificial neural network and tissue genotyping of hepatocellular carcinoma in liver-transplant recipients: Prediction of recurrence. Transplantation, 2005; 79 1737-40

37. Pommergaard HC, Burcharth J, Rosenberg J, Rasmussen A: Serologic and molecular biomarkers for recurrence of hepatocellular carcinoma after liver transplantation: A systematic review and meta-analysis. Transplant Rev (Orlando), 2016; 30: 171-77

38. Gruttadauria S, Tropea A, Pagano D et al: Mini-invasive approach contributes to expand the indication for liver resection for hepatocellular carcinoma without increasing the incidence of posthepatectomy liver failure and other perioperative complications: A single-center analysis. J Laparoendosc Adv Surg Tech A, 2016; 26: 439-46

39. Gruttadauria S, Pagano D, Tropea A et al: Laparoscopic approach for thermoablation microwave in the treatment of hepatocellular carcinoma: A single-center experience. J Laparoendosc Adv Surg Tech A, 2016; 26: 808-11

40. Gruttadauria S, di Francesco F, Spada M: Fifty-six-month survival after liver transplantation in a patient with more than one-hundred hepatocellular carcinoma nodules. Transpl Int, 2012; 9: e101-3 